\title{
No clinical benefit of vena cava filter in patients who can be anticoagulated
}

Inferior vena cava filters have increasingly been used in patients with acute venous thromboembolism over the past 3 decades, despite a lack of long-term data on their benefits and risks. A new randomized, open-label, blinded end point trial called PREPIC2 has shown that placement of a retrievable inferior vena cava filter for 3 months provides no clinical benefit in patients with acute symptomatic pulmonary embolism who are receiving anticoagulation for $\geq 6$ months.

The rate of recurrent pulmonary embolism was similar for the two treatment groups at both the 3-month (3.0\% vs $1.5 \%$ for controls; $P=0.50)$ and 6-month (3.5\% vs $2.0 \%$ for controls; $P=0.54$ ) follow-up. The rates of recurrent deep vein thrombosis, major bleeding, and all-cause death also did not differ significantly between the two groups at either timepoint. Access site haematomas, filter thrombosis, and retrieval failure due to mechanical reasons occurred in $2.6 \%$, $1.6 \%$, and $5.7 \%$ of patients who received a filter, respectively.

The PREPIC2 investigators conclude that their study findings do not support the use of a retrievable inferior vena cava filter in patients who can be treated with anticoagulation. "We believe that the low rate of events observed in the control group ... [indicate] that modern management with fulldose anticoagulation therapy is likely [to be] very effective even in patients usually considered to be at high risk for recurrence," write the investigators, "rendering unnecessary additional therapy such as inferior vena cava filters.”

Bryony M. Mearns

Original article Mismetti, P. et al. Effect of a retrievable inferior vena cava filter plus anticoagulation vs

anticoagulation alone on risk of recurrent pulmonary

embolism: a randomized clinical trial. JAMA 313, 1627-1635

(2015) 